\title{
Incidence of Neonatal Abstinence Syndrome — 28 States, 1999-2013
}

\author{
Jean Y. Ko, PhD ${ }^{1}$; Stephen W. Patrick, $\mathrm{MD}^{2}$; Van T. Tong, $\mathrm{MPH}^{1}$; Roshni Patel, $\mathrm{MPH}^{1}$; Jennifer N. Lind, PharmD 3 ; Wanda D. Barfield, MD ${ }^{1}$
}

Neonatal abstinence syndrome (NAS) is a postnatal drug withdrawal syndrome that occurs primarily among opioidexposed infants shortly after birth, often manifested by central nervous system irritability, autonomic overreactivity, and gastrointestinal tract dysfunction (1). During 2000-2012, the incidence of NAS in the United States significantly increased $(2,3)$. Several recent publications have provided national estimates of NAS $(2,3)$; however, data describing incidence at the state level are limited. CDC examined state trends in NAS incidence using all-payer, hospital inpatient delivery discharges compiled in the State Inpatient Databases of the Healthcare Cost and Utilization Project (HCUP) during 1999-2013. Among 28 states with publicly available data in HCUP during 1999-2013, the overall NAS incidence increased 300\%, from 1.5 per 1,000 hospital births in 1999 , to 6.0 per 1,000 hospital births in 2013. During the study period, significant increases in NAS incidence occurred in 25 of 27 states with at least 3 years of data, with annual incidence rate changes ranging from 0.05 (Hawaii) to 3.6 (Vermont) per 1,000 births. In 2013, NAS incidence ranged from 0.7 cases per 1,000 hospital births (Hawaii) to 33.4 cases per 1,000 hospital births (West Virginia). The findings underscore the importance of statebased public health programs to prevent unnecessary opioid use and to treat substance use disorders during pregnancy, as well as decrease the incidence of NAS.

NAS is a postnatal withdrawal syndrome that comprises a constellation of symptoms in newborns, including central nervous system irritability (e.g., tremors, increased muscle tone, high-pitched crying, and seizures), gastrointestinal dysfunction (e.g., feeding difficulties), and temperature instability (1). Although other substances have been implicated, NAS is most often attributed to in utero opioid exposure. This exposure can result from maternal prescription opioid use, which has increased nationally in recent years $(2,4)$, nonmedical opioid use, or medication-assisted treatment, which is long-term treatment with a longer acting but less euphoric opioid under medical supervision for opioid use disorder. Data on long-term developmental outcomes related to opioid exposure during pregnancy and NAS are limited.

The State Inpatient Databases include de-identified administrative data from all hospital inpatient discharges in a given state, regardless of payer. Data from State Inpatient Databases are compiled by state partners and then translated into a uniform format as part of HCUP, which is sponsored by the Agency for Healthcare Research and Quality. This analysis includes data from 28 states* whose data for 1999-2013 were publicly available on HCUP's online central distributor (https://www.hcup-us.ahrq.gov/tech_assist/centdist.jsp). Consistent with previous methodology (2,3), in-hospital births were identified using International Classification of Diseases, Ninth Revision, Clinical Modification (ICD-9-CM) codes V30.X-V39.X ending in 00 or 01 (indicating single or multiple live born infants), among all hospital discharge records during 1999-2013. Discharge records that did not have a principal or secondary diagnosis code indicating a hospital birth, or that indicated a transfer from another acute care hospital or health care facility, were excluded. Cases of NAS were identified with ICD-9-CM code 779.5 (drug withdrawal syndrome in a newborn). Cases of possible iatrogenic withdrawal, resulting from complications related to prolonged neonatal intensive care stay and not exposure during the antenatal period (ICD-9-CM codes: 765.01-765.05, 770.7, 772.1X, 779.7, 777.5X, 777.6), were excluded from the numerator.

Total incidence rates of NAS (cases per 1,000 births) were calculated for 1999 and 2013, using data available from 14 and 21 states, respectively. In addition, incidence rates of NAS were calculated for each state and year with available data during 1999-2013. Linear trends were assessed using logistic regression with NAS incidence as the outcome variable and infant birth year as the independent variable for the 27 states with at least 3 years of data. Annual incidence rate changes, which reflect average annual change in the incidence rate of NAS over time, were calculated from the beta coefficient of the infant's birth year with a state-specific intercept for states with significant linear trends. P-values $<0.05$ were considered to be statistically significant.

During 1999-2013, among 29,944,574 hospital births that occurred in the 28 states included in this report, 74,576 NAS cases occurred, reflecting an overall incidence rate of 2.5 cases per 1,000 hospital births. In 1999 (the first year), 2,419 NAS cases were identified among 1,610,733 births in 14 reporting states (1.5 per 1,000 births). In 2013, 8,270 NAS cases were identified among 1,385,371 births in 21 states ( 6.0 per 1,000 births).

Data for at least 5 consecutive years were available for 27 states (Table). In 1999, NAS incidence ranged from

\footnotetext{
*Arizona, Arkansas, California, Colorado, Florida, Hawaii, Iowa, Kentucky, Maine, Maryland, Massachusetts, Michigan, Mississippi, Nebraska, Nevada, New Jersey, New Mexico, New York, North Carolina, Oregon, Rhode Island, South Carolina, South Dakota, Utah, Vermont, Washington, West Virginia, and Wisconsin.
} 
TABLE. Neonatal abstinence syndrome (NAS) incidence rates per 1,000 hospital births,* by state and year — State Inpatient Databases, Healthcare Cost and Utilization Project, 1999-2013 ${ }^{\dagger}$

\begin{tabular}{|c|c|c|c|c|c|c|c|c|c|c|c|c|c|c|c|c|}
\hline \multirow[b]{2}{*}{ State } & \multicolumn{15}{|c|}{ Year } & \multirow{2}{*}{$\begin{array}{l}\text { Annual change } \\
\text { in incidence } \\
\text { rate }^{\S}\end{array}$} \\
\hline & 1999 & 2000 & 2001 & 2002 & 2003 & 2004 & 2005 & 2006 & 2007 & 2008 & 2009 & 2010 & 2011 & 2012 & 2013 & \\
\hline Arizona & 1.3 & 1.0 & 1.0 & 1.0 & 1.1 & 1.3 & 1.1 & 1.0 & 1.2 & 1.5 & 1.7 & 2.6 & 3.6 & 3.6 & 3.9 & 0.2 \\
\hline Arkansas & - & - & - & - & - & 0.4 & 0.9 & 0.4 & 0.7 & 1.0 & 1.3 & 1.4 & 2.2 & 2.7 & 2.6 & 0.3 \\
\hline California & 1.2 & 1.2 & 1.2 & - & 1.1 & 1.1 & 1.1 & 1.0 & 1.0 & 1.1 & 1.2 & 1.3 & 1.3 & - & $-\pi$ & $-\pi$ \\
\hline Colorado & 0.4 & 0.6 & 0.6 & 0.7 & 0.6 & 0.9 & 0.9 & 0.9 & 1.3 & 1.3 & 1.4 & 2.3 & 2.5 & 2.8 & 2.9 & 0.2 \\
\hline Florida & 0.4 & 0.4 & 0.5 & 0.6 & 0.7 & 0.7 & 0.9 & 1.3 & 1.7 & 2.3 & 3.4 & 4.9 & 5.9 & 5.9 & 6.3 & 0.6 \\
\hline Hawaii & - & 0.1 & 0.4 & 0.1 & 0.4 & 0.6 & - & 0.6 & 0.5 & 0.7 & 0.5 & 0.7 & 0.8 & 0.8 & 0.7 & 0.05 \\
\hline lowa & 0.3 & 0.4 & 0.3 & 0.5 & 0.6 & 0.4 & 0.4 & 0.5 & 0.6 & 0.7 & 0.8 & 1.1 & 1.3 & 1.9 & 2.2 & 0.1 \\
\hline Kentucky & - & 0.4 & 0.9 & 1.3 & 2.0 & 2.4 & 2.5 & 3.3 & 3.8 & 4.7 & 6.4 & 7.8 & 10.5 & 12.3 & 15.0 & 1.3 \\
\hline Maine & 1.1 & 0.9 & 2.0 & 3.0 & 5.2 & - & -9 & -9 & 12.6 & 15.5 & 19.0 & 21.5 & 21.3 & 30.4 & — & 3.0 \\
\hline Maryland & 7.6 & 6.5 & 7.1 & 6.7 & 6.3 & 6.2 & 6.6 & 6.6 & 6.5 & 7.1 & 8.2 & 9.5 & 10.6 & 11.4 & - & 0.3 \\
\hline Massachusetts & 2.2 & 2.5 & 2.7 & 2.6 & 2.9 & 3.8 & 4.4 & 5.0 & 6.1 & 6.7 & 8.5 & 10.0 & 10.8 & 12.5 & - & 0.9 \\
\hline Michigan & - & 0.4 & 0.5 & 0.6 & 0.8 & 0.9 & 1.2 & 1.2 & 1.7 & 2.0 & 2.9 & 3.6 & 5.0 & 5.4 & 6.7 & 0.6 \\
\hline Mississippi & - & - & - & - & - & -9 & - & -9 & - & - & - & 1.2 & 1.3 & —ा & - & - \\
\hline Nebraska & -9 & - & 0.2 & 0.1 & 0.3 & 0.2 & 0.7 & 0.4 & 0.2 & 0.4 & 0.7 & 1.0 & 0.8 & 1.4 & 1.6 & 0.1 \\
\hline Nevada & -9 & - & -9 & 1.1 & 0.8 & 1.3 & 1.5 & 1.2 & 1.3 & 1.6 & 2.0 & 3.0 & 3.3 & 5.0 & 4.8 & 0.4 \\
\hline New Jersey & 3.3 & 3.2 & 3.5 & 3.0 & 3.1 & 2.6 & 2.9 & 2.5 & 2.7 & 2.8 & 3.4 & 4.1 & 4.6 & 5.0 & 5.2 & 0.1 \\
\hline New Mexico & - & $-\pi$ & - & - & - & -9 & - & - & $-\pi$ & - & 3.7 & 4.2 & 5.8 & 7.8 & 8.5 & 1.5 \\
\hline New York & 1.4 & 1.5 & 1.3 & 1.2 & 1.2 & 1.3 & 1.2 & 1.2 & 1.4 & 1.5 & 1.8 & 1.9 & 2.6 & 2.8 & 3.6 & 0.1 \\
\hline North Carolina & -9 & 0.3 & 0.4 & 0.7 & 0.7 & 0.9 & 1.3 & 1.3 & 1.6 & 1.7 & 2.7 & 3.5 & 4.2 & 5.3 & 6.4 & 0.6 \\
\hline Oregon & 1.0 & 1.0 & 1.2 & 1.5 & 1.3 & 2.1 & 2.1 & 2.0 & 2.0 & 2.3 & 2.9 & 3.7 & 4.4 & 4.5 & 5.0 & 0.3 \\
\hline Rhode Island & - & - & -9 & 2.8 & 3.3 & 3.5 & 4.6 & 3.4 & 5.1 & 5.6 & 6.0 & 5.8 & 8.1 & 7.3 & - & 0.5 \\
\hline South Carolina & 1.3 & 0.9 & 0.6 & 0.4 & 0.7 & 1.1 & 1.1 & 1.5 & 1.3 & 1.5 & 1.9 & 2.2 & 2.7 & 3.3 & 3.9 & 0.2 \\
\hline South Dakota & $-\pi$ & -9 & -9 & - & - & -9 & - & -9 & 0.2 & 0.9 & 0.8 & 1.0 & 1.3 & 0.8 & 0.8 & - \\
\hline Utah & -9 & 0.8 & 0.9 & 1.3 & 1.0 & 1.3 & 2.0 & 1.9 & 2.3 & 2.5 & 2.5 & 3.5 & 4.1 & - & $-\pi$ & 0.3 \\
\hline Vermont & -9 & - & 0.7 & 2.7 & 3.7 & 4.1 & 8.1 & 9.1 & 12.5 & 15.8 & 20.9 & 25.3 & 26.2 & 30.5 & 33.3 & 3.6 \\
\hline Washington & 1.5 & 1.3 & 1.6 & 1.7 & 2.1 & 2.7 & 3.5 & 3.4 & 3.2 & 3.6 & 4.5 & 5.6 & 6.7 & 6.9 & 7.9 & 0.5 \\
\hline West Virginia & — & 0.5 & 1.0 & 1.7 & 3.3 & 3.4 & 6.9 & 7.1 & 7.5 & 10.2 & 11.0 & 14.2 & 16.9 & 21.7 & 33.4 & 2.7 \\
\hline Wisconsin & 0.4 & 0.4 & 0.3 & 0.5 & 0.6 & 1.1 & 0.9 & 1.7 & 1.9 & 2.5 & 2.9 & 4.1 & 5.5 & 5.7 & 7.9 & 0.7 \\
\hline
\end{tabular}

* Incidence rate numerator consisted of NAS cases excluding cases of iatrogenic withdrawal; incidence rate denominator consisted of state in-hospital births excluding transfers from another acute care hospital or healthcare facility.

+ Linear trends were assessed using logistic regression model for states with at least 3 years of data; $p$-values for linear trends were significant at $<0.05$ for all states except California and South Dakota.

$\S$ Annual change in incidence rates per 1000 hospital births were only assessed for states that had a significant linear trend, assessed using logistic regression, and estimated from the beta coefficient of the infant's birth year with a state specific intercept.

"Data not available or annual change in incidence rates not calculated because of insufficient data or nonsignificant linear trend.

0.3 per 1,000 births in Iowa to 7.6 per 1,000 births in Maryland. In 2013, NAS incidence ranged from 0.7 per 1,000 births in Hawaii to 33.4 per 1,000 births in West Virginia. During 2012-2013, three of 25 states (Maine, Vermont, and West Virginia), reported NAS incidence rates $>30$ per 1,000 hospital births (Figure). From 1999 to 2013, the incidence of NAS significantly increased in 25 of the 27 states with at least 3 years of data included in this report (Table). NAS incidence rates did not change significantly in California and South Dakota during 1999-2013. The annual incidence rate change over 1999-2013 was lowest in Hawaii (0.05 per 1,000 births) and highest in Vermont (3.6 per 1,000 births).

\section{Discussion}

The overall incidence of NAS in the states included in this report has increased almost 300\% during 1999-2013, from 1.5 to 6.0 cases per 1,000 hospital births. This increase in NAS incidence is consistent with that reported by other studies, which have described a national increase in incidence of $383 \%$ (from 1.2 cases per 1,000 hospital births in 2000 to 5.8 cases per 1,000 hospital births in 2012)(2,3). Substantial variation in NAS incidence and trends by state exist, with incidences in 2013 ranging from 0.7 (Hawaii) to 33.4 per 1,000 births (West Virginia). Differences in NAS incidence might be caused by variations across states in opioid prescribing rates (5), prevalence of illicit opioid use, or use of the ICD-9 code that identifies NAS.

State governments partially finance and fully administer Medicaid programs, direct block-grant funds relevant to treatment of substance use disorders and maternal and child health programs, and license health care professionals. Previous research indicates that Medicaid programs were financially responsible for approximately $80 \%$ of the estimated $\$ 1.5$ billion in NASrelated annual hospital charges in 2012 (3). Taken together, these factors make state-specific NAS estimates important to the formulation of public health plans to improve the health of mothers and infants affected by opioid use. 
FIGURE. Neonatal abstinence syndrome (NAS) incidence rate* 25 states, 2012-2013 ${ }^{\dagger}$

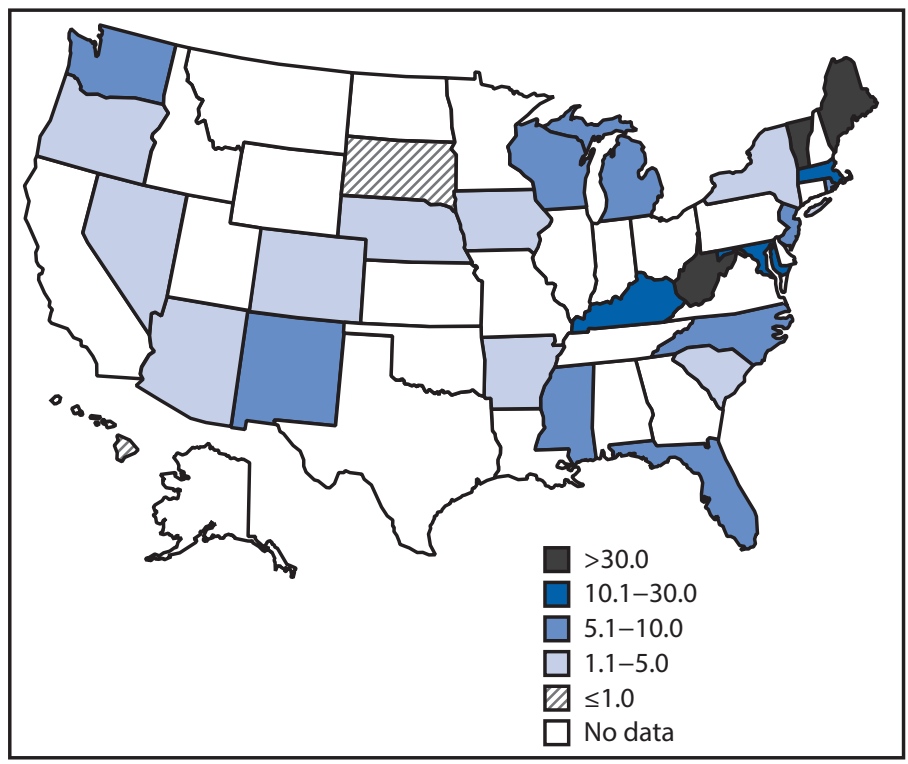

Source: State Inpatient Databases, Healthcare Cost and Utilization Project.

* NAS cases per 1,000 hospital births.

† Incidence rates reported are for 2013, except for four states (Maine, Maryland, Massachusetts, and Rhode Island) for which 2013 data were not available; 2012 data are reported for these states.

\section{Summary}

What is already known about this topic?

Neonatal abstinence syndrome (NAS) is a postnatal drug withdrawal syndrome in newborns caused primarily by in utero exposure to opioids. In the United States, the incidence of NAS increased $383 \%$ during 2000-2012, and an estimated $80 \%$ of hospital charges for NAS are covered by state Medicaid programs.

What is added by this report?

During 1999-2013, state-specific NAS incidence rates increased significantly in 25 of 27 states with at least 3 years of data, with annual changes in incidence rates ranging from 0.05 (Hawaii) to 3.6 (Vermont) per 1,000 hospital births. In 2013, NAS incidence ranged from 0.7 (Hawaii) to 33.4 cases (West Virginia) per 1,000 hospital births.

What are the implications for public health practice?

Prevention efforts, such as promotion of effective use of prescription drug monitoring programs, are needed to reduce inappropriate prescribing and dispensing of opioids. Clinicians should follow recommended guidelines on appropriate prescribing of opioid medications and provide screening and treatment for opioid use disorder among pregnant and nonpregnant women of reproductive age. Monitoring statespecific NAS incidence rates is important to ensure that adequate treatment and resources exist to address the effects of maternal opioid use and NAS within jurisdictions.
The findings in this report are subject to at least four limitations. First, the State Inpatient Databases include de-identified administrative data, and counts of NAS cases are based on information collected at the delivery hospitalization. In this analysis, only cases at the originating hospitals were counted. Cases identified as transfers from another hospital were not counted, to minimize possible duplication of counts and thus, overreporting of infants with NAS who might need a higher level of care. However, these rates are likely underestimates, as hospital administrative data identify fewer cases of NAS than does clinical reporting (๑). Second, these estimates are not generalizable to births that occur outside of the hospital; however, out-of-hospital deliveries represented only $1.5 \%$ of births in 2014 ( 7 ). Third, although statistically significant annual changes in incidence rates were observed, these changes might not represent large increases in actual numbers of affected infants, depending on the birth population in each state. Finally, data are not generalizable to the entire United States, but only to the 28 states included in this report.

Primary prevention measures are important in curbing the incidence of NAS. In 2016, CDC released the Guideline for Prescribing Opioids for Chronic Pain, which recommends that clinicians 1) consider nonopioid pharmacologic therapy for chronic pain management, 2) discuss family planning and how long-term opioid use might affect future pregnancies before initiating opioid therapy in reproductive-aged women, and $3)$ prescribe the lowest effective dose when opioids are started (8). Individual states have implemented strategies to address the opioid epidemic and NAS. Prescription drug monitoring programs are operational or will be implemented in 49 states and the District of Columbia (www.cdc.gov/drugoverdose/ pdmp) to track prescribing and dispensing of controlled prescription drugs; these programs have been shown to reduce inappropriate prescribing and overdose deaths (9). In addition, Florida, Georgia, Kentucky, and Tennessee have made NAS a reportable condition to state health departments to improve public health surveillance. Implementation of this type of passive surveillance of NAS can help states successfully target prevention and treatment measures, including access to medication-assisted treatment, the standard of care recommended by the American College of Obstetricians and Gynecologists for pregnant women with opioid use disorders (10). As part of the Protecting Our Infants Act of 2015, ${ }^{\dagger}$ CDC continues to provide technical assistance to states and American Indian tribes to improve NAS surveillance and to support implementation of effective public health measures.

$\bar{\dagger}$ https://www.congress.gov/bill/114th-congress/senate-bill/799. 


\section{Acknowledgments}

Mary D. Brantley, Division of Reproductive Health, National Center for Chronic Disease Prevention and Health Promotion, CDC; states participating in the State Inpatient Databases, Healthcare Cost and Utilization Project; Agency for Healthcare Research and Quality.

${ }^{1}$ Division of Reproductive Health, National Center for Chronic Disease Prevention and Health Promotion, CDC; ${ }^{2}$ Departments of Pediatrics and Health Policy, Division of Neonatology, Vanderbilt University School of Medicine, Nashville, Tennessee; ${ }^{3}$ Division of Congenital and Developmental Disorders, National Center on Birth Defects and Developmental Disabilities, CDC.

Corresponding author: Jean Y. Ko, JeanKo@cdc.gov, 770-488-5200.

\section{References}

1. Hudak ML, Tan RC; Committee on Drugs; Committee on Fetus and Newborn; American Academy of Pediatrics. Neonatal drug withdrawal. Pediatrics 2012;129:e540-60. http://dx.doi.org/10.1542/peds.2011-3212

2. Patrick SW, Schumacher RE, Benneyworth BD, Krans EE, McAllister JM, Davis MM. Neonatal abstinence syndrome and associated health care expenditures: United States, 2000-2009. JAMA 2012;307:193440. http://dx.doi.org/10.1001/jama.2012.3951

3. Patrick SW, Davis MM, Lehmann CU, Cooper WO. Increasing incidence and geographic distribution of neonatal abstinence syndrome: United States 2009 to 2012. J Perinatol 2015;35:650-5. http://dx.doi. org/10.1038/jp.2015.36
4. Desai RJ, Hernandez-Diaz S, Bateman BT, Huybrechts KF. Increase in prescription opioid use during pregnancy among Medicaid-enrolled women. Obstet Gynecol 2014;123:997-1002. http://dx.doi. org/10.1097/AOG.0000000000000208

5. Paulozzi LJ, Strickler GK, Kreiner PW, Koris CM. Controlled substance prescribing patterns-Prescription Behavior Surveillance System, eight states, 2013. MMWR Surveill Summ 2015;64(No. SS-9). http://dx.doi. org/10.15585/mmwr.ss6409a1

6. Burns L, Mattick RP. Using population data to examine the prevalence and correlates of neonatal abstinence syndrome. Drug Alcohol Rev 2007;26:487-92. http://dx.doi.org/10.1080/09595230701494416

7. Hamilton BE, Martin JA, Osterman MJK, Curtin SC, Mathews TJ. Births: final data for 2014. National vital statistics reports, vol. 64, no. 12. Hyattsville, MD: National Center for Health Statistics; 2015. http://www.cdc.gov/nchs/data/nvsr/nvsr64/nvsr64_12.pdf

8. Dowell D, Haegerich TM, Chou R. CDC guideline for prescribing opioids for chronic pain-United States, 2016. MMWR Recomm Rep 2016;65(No, RR-1). http://dx.doi.org/10.15585/mmwr.rr6501e1

9. Johnson H, Paulozzi L, Porucznik C, Mack K, Herter B. Decline in drug overdose deaths after state policy changes-Florida, 2010-2012. MMWR Morb Mortal Wkly Rep 2014;63:569-74.

10. American College of Obstetricians and Gynecologists. Opioid abuse, dependence, and addiction in pregnancy. Committee Opinion No. 524 Obstet Gynecol 2012;119:1070-6. http://dx.doi.org/10.1097/ AOG.0b013e318256496e 